\title{
ON ELECTRON ATTRACTION IN THE DIATOMIC BOND*
}

\author{
by
}

\section{Donald Greenspan}

Department of Mathematics

The University of Texas at Arlington

Technical Report \#284

Abstract
Previously, it was shown that classical simulation of the ground state diatomic hydrogen,
deuterium and tritium molecules yielded the correct vibrational frequencies and average molecular
diameters under the assumption that the bonding electrons attracted, rather than repelled. It is shown
here that the assumption of electron attraction also yields the correct vibrational frequencies and
average molecular diameters for $\mathrm{Li}_{2}^{7}, \mathrm{~B}_{2}^{11}$ and $\mathrm{C}_{2}^{12}$.
$\begin{aligned} & \text { * Computations performed on the CRAY Y-MP at the University of Texas Center for High AT ARLINGTON } \\ & \text { Performance Computing. }\end{aligned}$




\section{Introduction}

In this paper, the molecular bond is modelled with the aid of an electron attraction assumption. Electron attraction is, of course, not unknown, since it is fundamental in the quantum mechanical theory of superconductivity [1]. Nevertheless, we have not made this assumption arbitrarily, since it was derived computationally in the following way. Previously [2], it was found that classical dynamical calculations for the ground state $\mathrm{H}_{2}^{1}$ molecule, using coulombic forces and spectroscopic data, yielded a vibrational frequency of $(2.20) 10^{14} \mathrm{H}$, which deviates extensively from the experimental result (1.318)10 ${ }^{14} \mathrm{H}$ [3]. However, since quantum mechanics imply that two electrons in the same orbital repel with an effective force which is less than that of full coulombic repulsion, we repeated the classical calculation, but decreased the repulsive electron force by the factor 0.9 . Assuming conservation of energy, we adjusted the initial velocities of the electrons accordingly. And, since conservation of energy is essential in the numerical procedure, we used a numerical method designed for just such a purpose [4]. The vibrational frequency then decreased to (2.13)10 ${ }^{14} \mathrm{H}$. Encouraged by this reduction, we proceeded in the above spirit to decrease electron repulsion until the factor 0.9 as reduced to 0.0001 , but the vibrational frequency decreased only to $(1.78) 10^{14} \mathrm{H}$. We then proceeded through zero to choose negative factors until the coulombic force between the electrons was multiplied by -1.0 , that is, until the force between the electrons was assumed to be fully attractive rather than repulsive. The resulting vibrational frequency was in complete agreement with experiment, as were the corresponding frequencies obtained thereafter for various diatomic combinations of hydrogen, deuterium and tritium [5]. But, most unexpectedly, the molecular diameters were also in complete agreement with experiment.

In this paper we will show how an electron attraction assumption in the ground state molecules $\mathrm{Li}_{2}^{7}, \mathrm{~B}_{2}^{11}$, and $\mathrm{C}_{2}^{12}$ yields not only correct vibrational frequencies, but also, and again unexpectedly, accurate molecular diameters.

For later convenience, we have recorded in Table 1 the bonding energies $D_{0}$, total energies $E$,

TABLE 1

\begin{tabular}{|c|c|c|c|c|c|}
\hline Molecule & $D_{0}\left(10^{12} \mathrm{erg}\right)$ & $E\left(10^{12} \mathrm{erg}\right)$ & $r^{*}(\AA)$ & $f\left(10^{14} \mathrm{H}\right)$ & $d(\AA)$ \\
\hline $\mathrm{Li}_{2}^{7}$ & 1.65006 & -653.43338 & 0.186 & 0.105 & 2.6720 \\
$\mathrm{~B}_{2}^{11}$ & 5.76720 & -2155.5454 & 0.114 & 0.315 & 1.5890 \\
$\mathrm{C}_{2}^{12}$ & 5.76720 & -3306.1435 & 0.091 & 0.492 & 1.3117 \\
\hline
\end{tabular}


average radii $r^{*}$ of first ring electrons, vibrational frequencies, and average diameters of the diatomic molecules to be considered $[3,6,7,8]$.

\section{Considerations for $\mathrm{Li}_{2}^{7}$}

Since $\mathrm{H}_{2}^{1}, \mathrm{H}^{1} \mathrm{H}^{2}, \mathrm{H}_{2}^{2}, \mathrm{H}^{1} \mathrm{H}^{3}$ and $\mathrm{H}_{2}^{3}$ have already been discussed [5], and since ground state $\mathrm{He}_{2}$ is unstable, let us turn attention first to $\mathrm{Li}_{2}$ and describe the related computational considerations in detail. Because spectroscopic data are available only for $\mathrm{Li}_{2}^{7}$ [3], it is this diatomic molecule we study.

The ground state energy of the $\mathrm{Li}^{7}$ atom is $-(325.89166) 10^{12}$ erg [8]. Since the binding energy $D_{0}$ of $\mathrm{Li}_{2}^{7}$ is (1.65006) $10^{12} \mathrm{erg}$, we assume most simplistically that the total energy $E$ of ground state $\mathrm{Li}_{2}^{7}$ is

$$
E=2(-325.89116) 10^{-12}+(-1.65006) 10^{-12}=-(653.43338) 10^{-12} \mathrm{erg} .
$$

Next, in ground state $\mathrm{Li}_{2}^{7}$, let us denote the nuclei by $P_{1}, P_{2}$ and the electrons by $P_{3}, P_{4}, P_{5}$, $P_{6}, P_{7}, P_{8}$. Assume first that the only forces of interaction are coulombic. In cgs units, for $i=1,2$, $\ldots, 8$, and at any time, let $P_{i}$ be located at $\vec{r}_{i}=\left(x_{i}, y_{i}, z_{i}\right)$, have velocity $\vec{v}_{i}=\left(\dot{x}_{i}, \dot{y}_{i}, \dot{z}_{i}\right)$, and have acceleration $\vec{a}_{i}=\left(\ddot{x}_{i}, \ddot{y}_{i}, \ddot{z}_{i}\right)$. The classical molecular mechanics type equations for the motion of the $P_{i}$ from given initial data are

$$
m_{i} \vec{a}_{i}=\sum_{\substack{j=1 \\ j \neq 1}}^{8} \frac{e_{i} e_{j}}{r_{i j}^{2}} \frac{\vec{r}}{r_{i j}}, \quad \mathrm{i}=1,2, \ldots, 8,
$$

in which $\vec{r}_{j i}$ is the vector from $P_{j}$ to $P_{i}, r_{i j}=\left\|r_{i j}\right\|$, and

$$
\begin{gathered}
e_{3}=e_{4}=e_{5}=e_{6}=e_{7}=e_{8}=-(4.8028) 10^{-10} \mathrm{esu} \\
e_{1}=e_{2}=-3 e_{3} \\
m_{3}=m_{4}=m_{5}=m_{6}=m_{7}=m_{8}=(9.1085) 10^{-28} \mathrm{gr} \\
m_{1}=m_{2}=7(16724) 10^{-28} \mathrm{gr} .
\end{gathered}
$$

We now consider an arrangement of $P_{1}-P_{8}$ which incorporates a maximum amount of symmetry. As shown in Figure $1, P_{1}$ and $P_{2}$ are the nuclei; $P_{3}$ and $P_{4}$ are the first ring electrons associated with $P_{1} ; P_{5}$ and $P_{6}$ are the first ring electrons associated with $P_{2}$; and $P_{7}$ and $P_{8}$ are the 
two bonding second ring electrons. For this configuration, we now assume that $P_{7}$ and $P_{8}$ attract, rather than repel. Thus, the term containing $e_{7} e_{8}$ in (2.2) is altered to $-e_{7} e_{8}$.

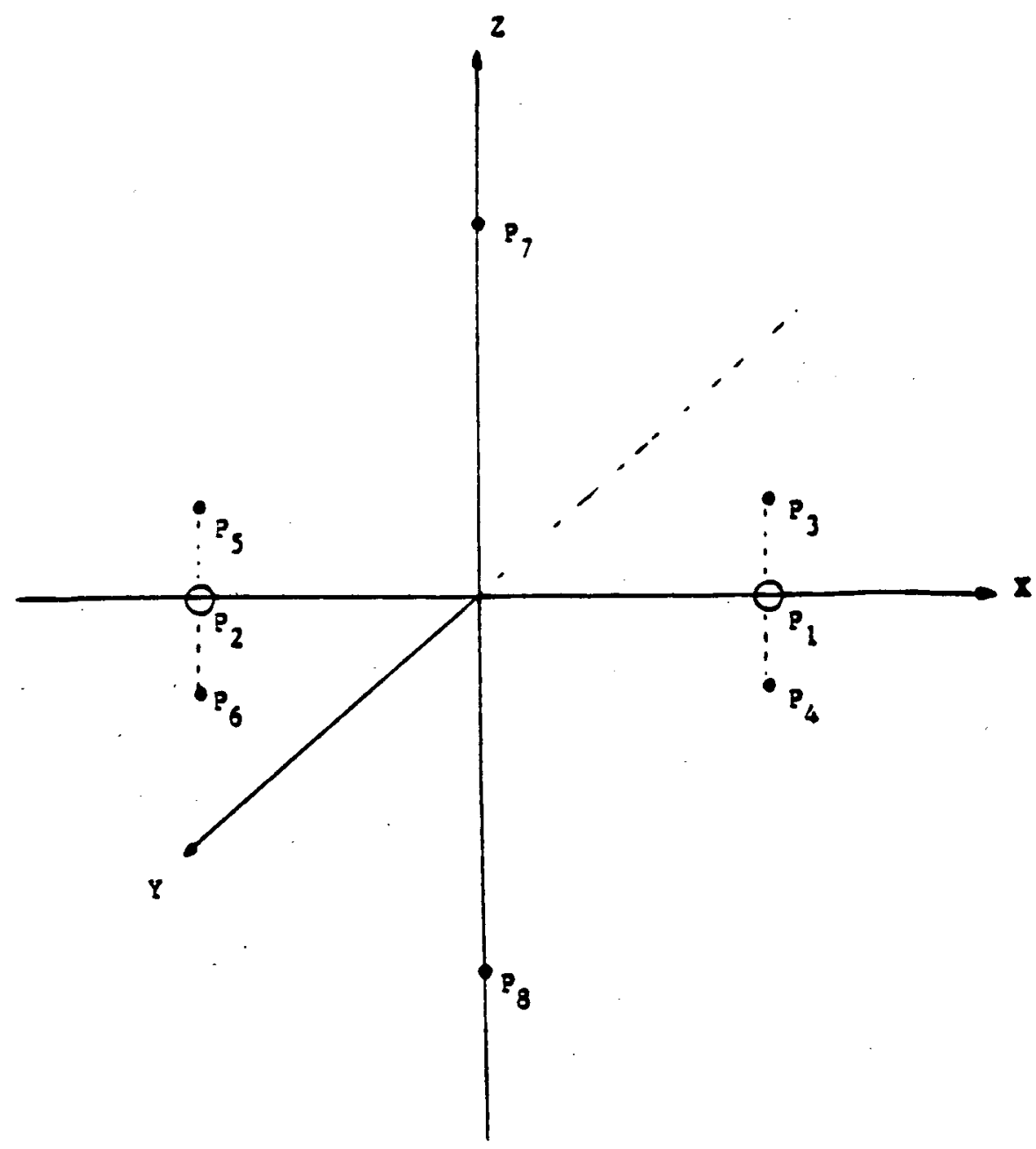

Figure 1.

For computational convenience, we make the changes of variables

$$
\vec{R}=(X, Y, Z)=10^{12}(x, y, z)=10^{12} \vec{r}
$$

$$
T=10^{22} t
$$


and define

$$
\vec{V}_{i}=\left(\frac{d X_{i}}{d T}, \frac{d Y_{i}}{d T}, \frac{d Z_{i}}{d T}\right)
$$

so that

$$
\vec{v}_{i}=10^{10} \vec{V}_{i}
$$

We now choose initial data in the $(X, Y, Z)$ variables, so that $\left(X_{i}, Y_{i}, Z_{i}\right)$ are the coordinaates of $P_{i}$. To allow for a variety of cases, $X_{1}$ and $Z_{7}$ are allowed to be parameters, while symmetry is imposed by choosing $X_{2}=-X_{1}, X_{3}=X_{4}=X_{1}, X_{5}=X_{6}=X_{2}, X_{7}=X_{8}=0 ; Y_{i}=0$ for all $i$; $Z_{1}=Z_{2}=0, Z_{3}=-Z_{4}=Z_{5}=-Z_{6}=1860, Z_{8}=-Z_{7}$. The choice 1860 follows from the known average electron radius $r^{*}$ in $\mathrm{Li}^{+}$(see Table 1). For initial velocities, we choose $\vec{V}_{1}=\vec{V}_{2}=\overrightarrow{0}$, $\vec{V}_{3}=-\vec{V}_{4}=\vec{V}_{5}=-\vec{V}_{6}=(0,0.06328,0), \vec{V}_{7}=-\vec{V}_{8}=\left(0, V_{y}, 0\right)$. The choice 0.06328 follows from energy conservation for ground state $L_{i}^{+}$, or, equivalently, from

$$
-(317.339) 10^{-4}=9.1085 V_{3}^{2}+23.06689(-6 / 1860+1 / 3720) \text {, }
$$

since the ground state energy of $\mathrm{Li}^{+}$is $-317.339\left(10^{-12}\right)$ erg [8].

The energy equation for ground state $\mathrm{Li}_{2}^{7}$, at any time, is equivalent to

$$
-(653.43338) 10^{-4}=(4.55425)\left(4 V_{3}^{2}+2 V_{7}^{2}\right)+(58534)\left(V_{1}^{2}+V_{2}^{2}\right)
$$

$+23.06689\left(9 / R_{12}-12 / R_{13}-12 / R_{15}-12 / R_{17}+2 / R_{34}+2 / R_{35}+2 / R_{36}+4 / R_{37}+4 / R_{38}-1 / R_{78}\right)$

In Table 2 are listed an arbitrary selection of $X_{1}$ and $Z_{7}$ values and the resulting velocity component $V_{y}$ for $P_{7}$, as determined from (2.12).

TABLE 2

\begin{tabular}{|c|r|r|r|c|c|}
\hline Cases & $X_{1}$ & $Z_{7}$ & $V_{y}$ & $f\left(10^{14} \mathrm{H}\right)$ & $d(\AA)$ \\
\hline 1 & 18000 & 19000 & 0.013263691 & 0.112 & 2.455 \\
2 & 20000 & 20000 & 0.012296058 & 0.110 & 2.572 \\
3 & 22500 & 22500 & 0.010569289 & 0.106 & 2.740 \\
4 & 25000 & 30000 & 0.006667666 & 0.104 & 2.892 \\
5 & 30000 & 35000 & 0.002733061 & 0.0959 & 3.310 \\
\hline
\end{tabular}




\section{Computational Results for $\mathrm{Li}_{2}^{7}$}

For $\mathrm{Li}_{2}^{7}$, energy conserving calculations were now implemented for solving differential system (2.2). (A FORTRAN computer program is available to the interested reader [9]). The extensive symmetry imposed allowed for the relatively large time step $\Delta T=3.0$, which was checked for accuracy periodically with $\Delta T=0.1$. The resulting frequencies and average diameters are recorded in the final two columns of Table 2. All the results are in good agreement with the experimental values $(0.105) 10^{14} \mathrm{H}$ and $2.672 \AA$. This is valid even though the $X_{1}$ and $Z_{7}$ choices vary extensively. The results of Cases 3 and 4 agree remarkably well with the experimental results. Repeating Cases 1-5, but with repulsion between $P_{7}$ and $P_{8}$ yields frequencies which deviate from experiment by more than $60 \%$.

Note that (2.11) constrains the positions that particles can have. The reason is that kinetic energy is always positive, so that one must always satisfy the relationship

$$
\begin{aligned}
& -(653.43338) 10^{-4}-23.06689\left(9 / R_{12}-12 / R_{13}-12 / R_{15}-12 / R_{17}+2 / R_{34}\right. \\
& \quad+2 / R_{35}+2 / R_{36}+4 / R_{37}-1 / R_{78}>0 .
\end{aligned}
$$

All of the entries in Table 2 satisfy this relationship. The choice $X_{1}=16000$ and $Z_{7}=40000$, however, does not. Indeed, the choices in Table 2 range fairly well over the allowable choices.

We now make a final, but important, observation about the computations of this section. Results entirely comparable to those of Table 2 were obtained more simply by using the $X_{1}, Z_{7}, V_{y}$ initial data in Table 2 and incorporating the first ring electrons into their respective nuclei. The masses of the nuclei are essentially the same, while their charges change to that of a single proton. Thereby, the problem reduces from an eight-body problem to a four-body problem. For the Case 2 data, the resulting frequency was $(0.106) 10^{14} \mathrm{H}$ and the diameter was $2.740 \AA$, while for the Case 3 data $(0.105) 10^{14} \mathrm{H}$ and $2.667 \AA$ resulted. Using electron repulsion again yielded large errors in the frequencies.

\section{Computational Results for $\mathrm{B}_{2}^{11}$}

Since $\mathrm{Be}_{2}$, like $\mathrm{He}_{2}$, is unstable, the next diatomic molecule of interest is $\mathrm{B}_{2}^{\mathbf{1 1}}$. The charge of

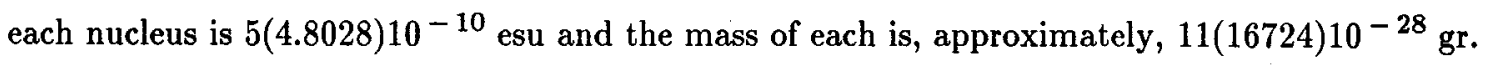

The complexity which is now faced is that there are six second ring electrons and their possible arrangements and interactions allow for many more possible configurations than was the case for $\mathrm{Li}_{2}^{7}$. We will then begin with the very particular arrangement shown in Figure 2, and later discuss a variety of other configurations which were studied. Note that for computational ease, the choice of axes in 
Figure 2 is different from that of Figure 1.

For $Z_{1}$ a positive parameter, let $P_{1}$ and $P_{2}$, the nuclei, be located initially at $\left(0,0, Z_{1}\right)$, $\left(0,0,-Z_{1}\right)$, respectively. From Table 1 , the inner electrons of a boron atom in ground state have radii $0.114 \AA$, so $P_{3}, P_{4}, P_{5}, P_{6}$ are set at $\left(1140,0, Z_{1}\right),\left(-1140,0, Z_{1}\right),\left(1140,0,-Z_{1}\right),(-1140,0$, $\left.-Z_{1}\right)$, respectively. For $X_{7}$ a parameter, let the position of $P_{7}$ be $\left(X_{7}, 0, Z_{1}\right)$.

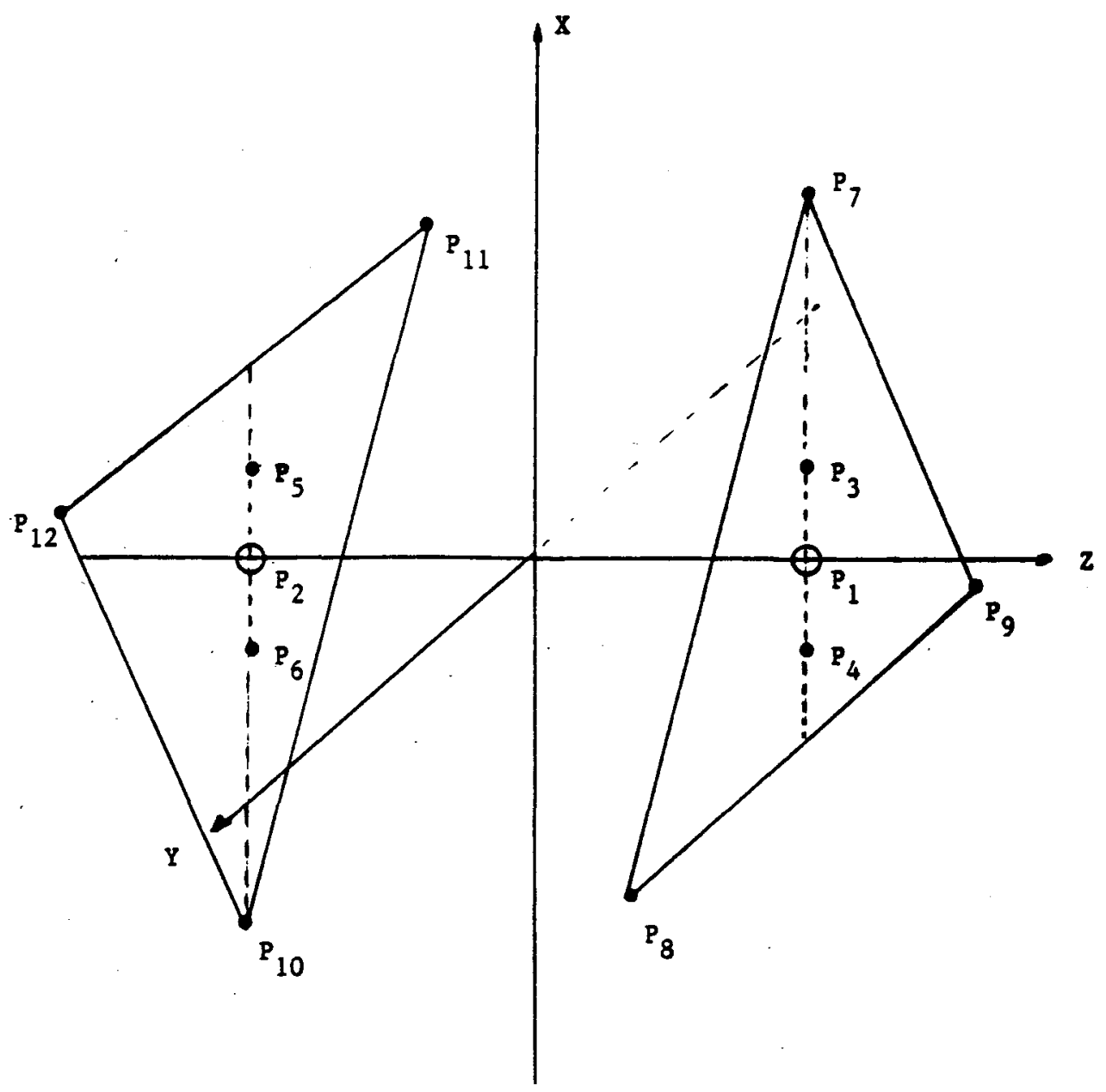

Figure 2.

$P_{8}$ and $P_{9}$ are then located at the vertices of a regular triangle which is parallel to the $X Y$ plane and has $P_{1}$ as its centroid. The coordinates of $P_{8}$ and $P_{9}$ are $\left(X_{8}, Y_{8}, Z_{8}\right)=\left(-0.5 X_{7}, 0.086602540 X_{7}\right.$, $\left.Z_{1}\right),\left(X_{9}, Y_{9}, Z_{9}\right)=\left(-0.5 X_{7},-0.86602540 X_{7}, Z_{1}\right) . P_{10}, P_{11}, P_{12}$ are set finally to be symmetric relative to the origin with $P_{7}, P_{8}, P_{9}$, respectively. 
For initial velocities, set $\vec{V}_{1}=\vec{V}_{2}=\overrightarrow{0}$. The speeds of $P_{3}-P_{6}$ are determined from ground state $\mathrm{B}^{3+}$, whose energy is $-(960.535) 10^{-12} \mathrm{erg}[8]$. The equation which now corresponds to (2.11) is

$$
-(960.535) 10^{-4}=(9.1085) V_{3}^{2}+23.06689(-10 / 1140+1 / 2280) \text {, }
$$

from which it follows that $V_{3}=0.10289$. Thus, we take $\vec{V}_{3}=\left(\begin{array}{llll}0, & 0.10289, & 0\end{array}\right)$ and $\vec{V}_{4}=-\vec{V}_{5}=\vec{V}_{6}=-\vec{V}_{3}$. The velocity vector $\vec{V}_{7}$ is taken to be $\left(0, V_{y}, 0\right)$, in which $V_{y}$ will be determined shortly. Once $\vec{V}_{7}$ is determined, we will set $\vec{V}_{8}=\left(-0.86602540 V_{y},-0.5 V_{y}, 0\right)$, $\vec{V}_{9}=\left(0.86602540 V_{y},-0.5 V_{y}, 0\right), \vec{V}_{10}=-\vec{V}_{7}, \vec{V}_{11}=-\vec{V}_{8}, \vec{V}_{12}=-\vec{V}_{9}$.

Next note that since the energy of a ground state boron atom is $-(1074.8891) 10^{-12} \mathrm{erg}[8]$, and, from Table 1, the bonding energy of $\mathrm{B}_{2}^{11}$ is $(5.76720) 10^{-12} \mathrm{erg}$, we assume that the total energy $E$ of $\mathrm{B}_{2}^{11}$ is

$$
E=2(-1074.8891) 10^{-12}-5.76720\left(10^{-12}\right)=-(2155.5454) 10^{-12} \mathrm{erg} .
$$

Now, were all interactions strictly coulombic, then the equations of motion for $P_{1}-P_{12}$ from given initial data would be

$$
m_{i} \vec{a}_{i}=\sum_{\substack{j=1 \\ j \neq i}}^{12} \frac{e_{i} e_{j}}{r_{i j}{ }^{2}} \frac{\vec{r}_{j i}}{r_{i j}}, \quad i=1,2, \ldots, 12
$$

and the energy equation for the system would be

$$
E=\frac{1}{2} \sum_{i=1}^{12} m_{i} v_{i}^{2}+\sum_{\substack{i, j=1 \\ i<j}}^{12} \frac{e_{i} e_{j}}{r_{i j}}
$$

In the $R, V$ notation, equations (4.2) and (4.4) imply

$$
-(2155.5454) 10^{-12}=\frac{1}{2}\left(10^{20}\right) \sum_{i=1}^{12} m_{i} V_{i}^{2}+10^{12} \sum_{\substack{i, j=1 \\ i<j}}^{12} \frac{e_{i}{ }^{e} j}{R_{i j}}
$$

We now assume that the pairs $\left(P_{7}, P_{10}\right),\left(P_{8}, P_{11}\right),\left(P_{9}, P_{12}\right)$ attract, rather than repel. Thus, equations (4.3) - (4.5) must be adjusted so that $e_{7} e_{10}, e_{8} e_{11}, e_{9} e_{12}$ are replaced by $-e_{7} e_{10},-e_{8} e_{11}$, $-e_{9} e_{12}$, respectively. When this is done, the initial velocity $\vec{V}_{7}$ can be determined directly from (4.5) for various choices of $Z_{1}$ and $X_{7}$. In Table 3, the value $V_{y}$ for $\vec{V}_{7}$ has been so determined for the given $Z_{1}$ and $X_{7}$ values. 
TABLE 3

\begin{tabular}{|c|c|c|c|c|c|}
\hline Cases & $Z_{1}$ & $X_{7}$ & $V_{y}$ & $f\left(10^{14} \mathrm{H}\right)$ & $d(\AA)$ \\
\hline 1 & 9500 & 7500 & 0.030849845 & 0.2884 & 1.4680 \\
2 & 10500 & 8500 & 0.027207169 & 0.3060 & 1.5446 \\
3 & 11000 & 8500 & 0.027137623 & 0.3151 & 1.5680 \\
4 & 11500 & 8000 & 0.028747770 & 0.3242 & 1.6018 \\
5 & 12000 & 8500 & 0.026993996 & 0.3351 & 1.6409 \\
\hline
\end{tabular}

The actual system simulation is now simplified, as indicated at the end of Section 3, by incorporating the first ring electrons $P_{3}-P_{6}$ into their respective nuclei, yielding a nuclear charge of $3(4.8028) 10^{-10}$ esu and resulting in a simpler eight-body problem in which the initial data for $P_{1}, P_{2}$, $P_{7}-P_{12}$ are exactly those positions and velocities determined from the full twelve-body considerations. For $\Delta T=2.0$, with accuracy checked periodically using $\Delta T=0.1$, the resulting frequencies are listed in Table 3 and are entirely in agreement with the experimental result $(0.315) 10^{14} \mathrm{H}$. Again, unexpectely, the resulting average diameters, also recorded in Table 3, are also in agreement with the experimental value (1.5890) $\AA$. Case 3 is the best of the five.

A very large number of configurations other than that shown in Figure 2 were also studied and the results are summarized as follows. Most of these had two, four, or six electrons in motion in the $X Y$ plane and allowed one pair, two pairs, or three pairs of electrons to be in an attraction mode. All of these were physically unstable and resulted in molecular degeneration, whether a twelve-body or an eight-body simulation was considered. These cases were the first ones to be studied and were motivated by the $\mathrm{Li}_{2}^{7}$ results and by Figure 1, but all proved to be incorrect. Cases in which triangle $P_{10} P_{11} P_{12}$ in Figure 2 was set symmetrically with triangle $P_{7} P_{8} P_{9}$ about the $X Y$ plane led to instability when both triangles were set in motion in the same direction. When these motions were opposite to each other, the nuclei seemed to move at random and the electrons would often interchange positions relative to the $X Y$ plane. Allowing different electron pairs to be in an attraction mode, other than those whose results are recorded in Table 2, never yielded correct frequency and diameter results simultaneously.

5. Computational Results for $\mathrm{C}_{2}^{12}$

For $\mathrm{C}_{2}^{12}$ the charge of each nucleus is $6(4.8028) 10^{-10}$ esu and the mass of each is, 


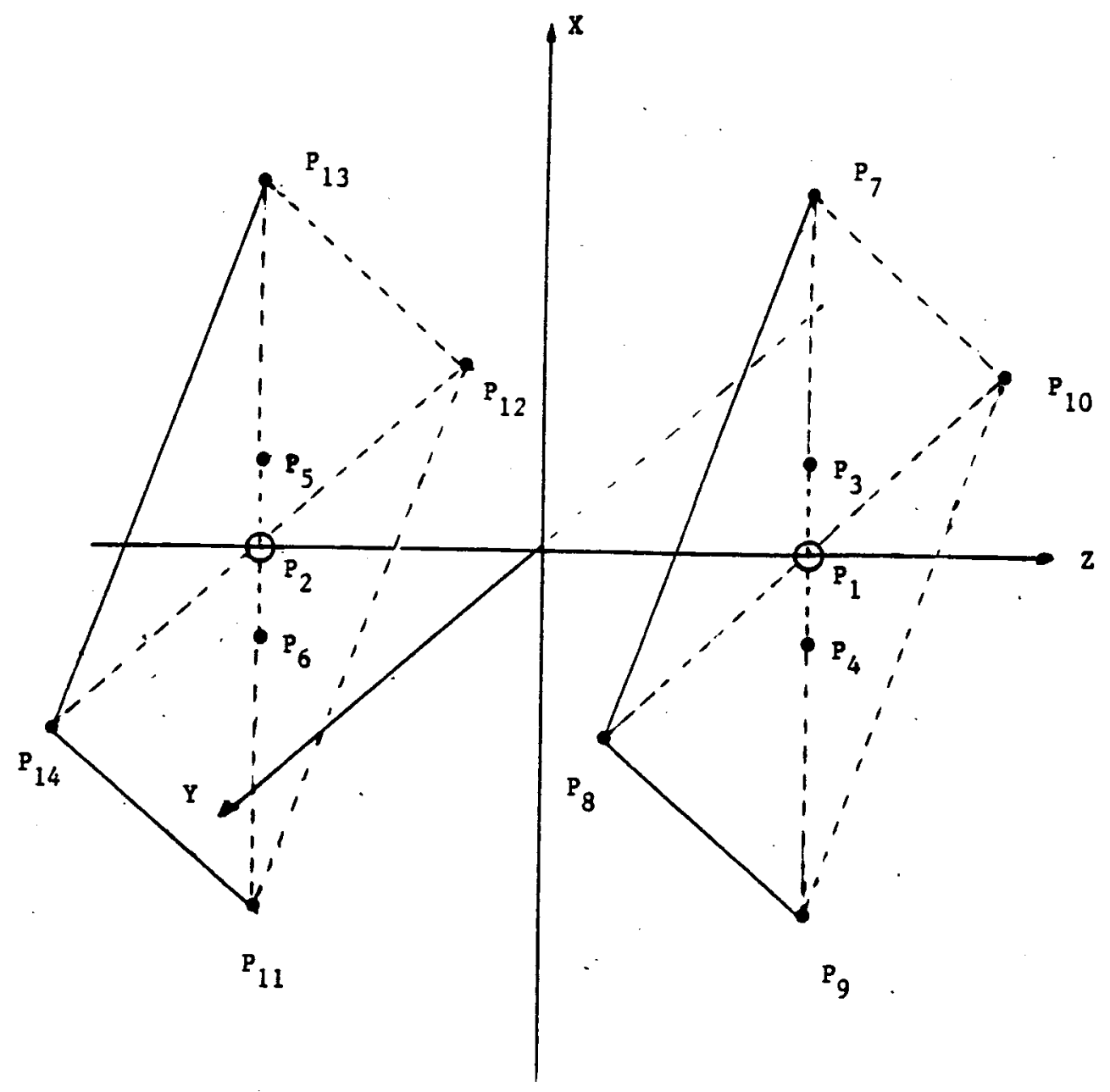

Figure 3.

approximately, $12(16724) 10^{-28} \mathrm{gr}$.

Motivated by the $\mathrm{B}_{2}^{11}$ results, in which diametrically opposite electrons in Figure 2 were in an attraction mode, we began with the symmetric $\mathrm{C}_{2}^{12}$ arrangement in Figure 3 and proceeded as follows. Let $P_{1}, P_{2}$ be the nuclei, located at $\left(0,0, Z_{1}\right),\left(0,0,-Z_{1}\right)$, respectively. From Table 1 , the inner electrons of a ground state carbon atom have radii $0.091 \AA$, so that $P_{3}, P_{4}, P_{5}, P_{6}$ are set at $\left(910,0, Z_{1}\right),\left(-910,0, Z_{1}\right),\left(910,0,-Z_{1}\right),\left(-910,0,-Z_{1}\right)$, respectively. The position of $P_{7}$ is taken to be $\left(X_{7}, 0, Z_{1}\right)$. For symmetry, $P_{8}, P_{9}, P_{10}, P_{11}, P_{12}, P_{13}, P_{14}$ are then set at $\left(0, X_{7}, Z_{1}\right)$, $\left(-X_{7}, 0, Z_{1}\right), \quad\left(0,-X_{7}, Z_{1}\right), \quad\left(-X_{7}, 0,-Z_{1}\right), \quad\left(0,-X_{7},-Z_{1}\right), \quad\left(X_{7}, 0,-Z_{1}\right), \quad\left(0,-X_{7},-Z_{1}\right)$, respectively.

For initial velocities, set $\vec{V}_{1}=\vec{V}_{2}=\overrightarrow{0}$. The speeds $V_{3}=V_{4}=V_{5}=V_{6}$ of $P_{3}-P_{6}$ are determined from consideration of ground state $\mathrm{C}^{4+}$, whose energy is $(-0.1413) 10^{-8} \mathrm{erg}$ [8], so that 


$$
-1413\left(10^{-4}\right)=9.1085 V_{3}^{2}+23.06689(-12 / 910+1 / 1820) \text {. }
$$

From (5.1), one finds $V_{3}=0.12842$. Thus, we set $(0,0.12842,0)=\vec{V}_{3}=-\vec{V}_{4}=\vec{V}_{5}=-\vec{V}_{6}$. Now set $\vec{V}_{7}=\left(0, V_{y}, 0\right)$, in which $V_{y}$ will be determined shortly. Once $V_{y}$ is determined, we then set $\vec{V}_{8}=\left(-V_{y}, 0,0\right), \quad \vec{V}_{9}=-\vec{V}_{7}, \quad \vec{V}_{10}=-\vec{V}_{8} . \quad$ For symmetry, we set $\vec{V}_{11}=-\vec{V}_{7}, \quad \vec{V}_{12}=-\vec{V}_{8}$, $\vec{V}_{13}=-\vec{V}_{9}, \vec{V}_{14}=-\vec{V}_{10}$. Let us then show how to determine $V_{y}$.

Since the ground state energy of a carbon atom is $-(1650.1882) 10^{-12} \mathrm{erg}[8]$, and, from Table 1, the binding energy of $\mathrm{C}_{2}^{12}$ is (5.7672)10-12 $\mathrm{erg}$, we assume that the total energy $E$ of $\mathrm{C}_{2}^{12}$ is

$$
E=-2(1650.1882) 10^{-12}-(5.7672) 10^{-12}=-(3306.1436) 10^{-12} \mathrm{erg} .
$$

Now, if all interactions are strictly coulombic, then the equations of motion for $P_{1}-P_{14}$ would be

$$
m_{i} \vec{a}_{i}=\sum_{\substack{j \neq 1 \\ j \neq i}}^{14} \frac{e_{i} e_{j}}{r_{i j}^{2}} \frac{\vec{r}_{j i}}{r_{i j}}, \quad i=1,2, \ldots, 14
$$

and the energy equation of the system would be

$$
E=\frac{1}{2} \sum_{i=1}^{14} m_{i} v_{i}^{2}+\sum_{\substack{i, j=1 \\ i<j}}^{14} \frac{e_{i}^{e} j}{r_{i j}}
$$

In the $R, V$ terminology, equations (5.2) and (5.4) imply

$$
-3306.1436\left(10^{-12}\right)=\frac{1}{2}\left(10^{20}\right) \sum_{i=1}^{14} m_{i} V_{i}^{2}+10^{12} \sum_{\substack{i, j=1 \\ i<j}}^{14} \frac{e_{i} e_{j}}{R_{i j}} .
$$

Assume now that the pairs $\left(P_{7}, P_{11}\right),\left(P_{8}, P_{12}\right),\left(P_{9}, P_{13}\right),\left(P_{10}, P_{14}\right)$, that is, the diametrically opposite electron pairs, attract rather than repel. This is similar to the assumption for $B_{2}^{11}$ in the sense that pairs of electrons which are separated maximally attract. Thereby, equations (5.3) - (5.5) must be adjusted so that $e_{7} e_{11}, e_{8} e_{12}, e_{9} e_{13}, e_{10} e_{14}$ are replaced by $-e_{7} e_{11},-e_{8} e_{12},-e_{9} e_{13},-e_{10} e_{14}$, respectively. When this is done, the parameter $V_{y}$ can be determined directly from (5.5) for various choices of $Z_{1}$ and $X_{7}$. In Table 4, the value $V_{y}$ has been determined in this fashion for the given $Z_{1}$ and $X_{7}$ values. Note again that a constraint similar to $(3.1)$ is valid, so that, for example, the choice $Z_{1}=8000, X_{7}=14000$ is not possible, since it yields an imaginary value for $V_{y}$.

The motion of the system is simulated by incorporating the first ring electrons into their respective nuclei, yielding a nuclear charge of $4(4.8028) 10^{-10} \mathrm{esu}$, and resulting in a ten-body problem in which the initial data for $P_{1}, P_{2}, P_{7}-P_{14}$ are exactly those determined by the full fourteen-body 
TABLE 4

\begin{tabular}{|c|c|c|c|c|c|}
\hline Case & $Z_{1}$ & $X_{7}$ & $V_{y}$ & $f\left(10^{14} \mathrm{H}\right)$ & $d(\AA)$ \\
\hline 1 & 7000 & 6000 & 0.038655171 & 1.883 & 1.2750 \\
2 & 7500 & 8000 & 0.028248164 & 1.951 & 1.3047 \\
3 & 8000 & 7000 & 0.033111460 & 1.974 & 1.3127 \\
4 & 8500 & 6000 & 0.038406517 & 2.027 & 1.3413 \\
5 & 9000 & 8000 & 0.028228198 & 2.115 & 1.3819 \\
6 & 9500 & 9000 & 0.032909247 & 2.182 & 1.4030 \\
7 & 8000 & 10000 & 0.019113853 & 2.113 & 1.4587 \\
\hline
\end{tabular}

problem. For $\Delta T=2.0$, with acuracy checked periodically with $\Delta T=0.1$, the resulting frequencies and diameters are recorded in Table 4. Again, both frequencies and diameters agree with the experimental results $f=(2.031) 10^{14} \mathrm{H}, d=(1.3117) \AA$.

A variety of other configurations and initial conditions were studied, but none yielded correct results for both the frequency and the diameter simultaneously. These cases included increasing the number of attracting pairs, changing the pairs which attracted, and rotating $P_{10}-P_{14}$ through a $45^{\circ}$ angle.

\section{Concluding Remarks.}

Under the assumption that electron pairs can attract, we have shown that a deterministic dynamical simulation of electron and nuclei motions yield correct vibrational frequencies and molecular diameters for ground state $\mathrm{Li}_{2}^{7}, \mathrm{~B}_{2}^{11}$ and $\mathrm{C}_{2}^{12}$. Each case was run under the assumption that the initial velocities of the nuclei were zero, which is not necessarily correct, and yet all the results were good, and at least one result was excellent in all respects. It was probably for the excellent results that the assumption of zero initial velocity for the nuclei was most nearly correct.

The models were all ultrasimplistic and improvement should be made to include magnetic effects and classical spin.

Unfortunately, we do not know as yet how to introduce excited states, important as they are.

Finally, note that we feel that a possible subquark structure of the electron [10] and possible quark structures of nuclei [11] may relate to our assumption of electron attraction. 


\section{REFERENCES}

1. J. Bardeen, L. N. Cooper, and J. R. Schrieffer, "Theory of superconductivity," Phys. Rev., 108, 1957, p. 1175.

2. D. Greenspan, "On the error in quasi-quantum mechanical calculations," Comp. Math. Applic., 22,1991 , p. 73.

3. G. Herzberg, Molecular Spectra and Molecular Structure, 2nd Ed., van Nostrand, NY, 1965, pp. $3,506,513,532,546$.

4. D. Greenspan, Arithmetic Applied Mathematics, Pergamon, Oxford, 1980, p. 13.

5. D. Greenspan, "On electron attraction," Molec. Phys.

6. J. T. Waber and D. T. Cromer, "Orbital radii of atoms and ions," J. Chem. Phys., 42, 1965, p. 4118 .

7. D. Greenspan, "Classical computer studies of one-electron and two-electron atoms and ions," TR 282, Dept. Math., Univ. Texas at Arlington, 1991.

8. David R. Lide (ed.), CRC Handbook of Chemistry and Physics, CRC Press, Boca Raton, FL, 1990-1991, p. 10-201.

9. D. Greenspan, "On electron attraction in the diatomic bond," TR 284, Dept. Math., Univ. Texas at Arlington, 1991.

10. H. Dehmelt, "Experiments in the structure of an individual elementary particle," Science, 247, 1990 , p. 539.

11. S. Weinberg, The Discovery of Subatomic Particles, Freeman, NY, 1990, p. 156. 